\title{
Utilização de regressão logística simples na verificação da qualidade do ar atmosférico de Uberlândia
}

\author{
Use of simple logistic regression when checking the atmospheric air \\ quality in Uberlândia
}

\author{
Renata Carvalho Macedo Leite \\ Acadêmica do Curso de Ciências Biológicas do Instituto de Biologia da Universidade Federal de Uberlândia (UFU) \\ Ednaldo Carvalho Guimarães \\ Doutor. Professor Associado da Faculdade de Matemática da UFU \\ Euclides Antonio Pereira de Lima \\ Doutor em Engenharia Química pela Faculdade de Engenharia Química da UFU \\ Marcos Antonio de Souza Barrozo \\ Doutor. Professor Associado da Faculdade de Engenharia Química da UFU
}

Marcelo Tavares

Doutor. Professor Associado da Faculdade de Matemática da UFU

\section{Resumo}

O objetivo desta pesquisa foi analisar a qualidade do ar atmosférico de Uberlândia, em Minas Gerais, por meio de modelos de regressão logística simples. O equipamento utilizado na obtenção dos dados de poluição foi o amostrador de partículas suspensas menores que $10 \mu \mathrm{m}\left(\mathrm{MP}_{10}\right)$. As medidas de concentração do material particulado foram realizadas no terminal central de ônibus urbanos de Uberlândia. Neste trabalho, foram utilizados os dados do período de 2003 a 2008. As variáveis preditoras referentes ao clima (umidade relativa, velocidade do vento, precipitação diária e temperatura média) e o fluxo de veículos foram utilizadas de forma contínua no modelo logístico. Já as variáveis relacionadas ao dia da semana e à estação do ano foram codificadas de forma binária. Os resultados revelaram que existe uma relação significativa das variáveis temporais e climáticas com a qualidade do ar de Uberlândia. Os modelos logísticos simples mostraram que a precipitação, a umidade relativa, o verão, a primavera, o sábado e o domingo contribuem significativamente para se obter qualidade do ar boa, enquanto o inverno e o fluxo de veículos crescente tendem a piorar a qualidade do ar.

Palavras-chave: particulados no ar; $\mathrm{MP}_{10}$; modelo logístico.

\section{Abstract}

The objective of this paper was to analyze the air quality in Uberlândia, in Minas Gerais, by simple logistic regression models. The PM $_{10}$ sampler was used to obtain data of the pollution that considers only concentration of suspended particles smaller than $10 \mu \mathrm{m}$. The temperature, precipitation, relative humidity, wind speed, day of the week and seasons of year were used as predictions variables in this study. The day of week and seasons of year were used as binary system and as predictions variables too. The results showed that there is a significant relation among the temporal and climatic variables with the air quality of Uberlândia. The simple logistic models show that precipitation, humidity, summer, spring, Saturday, and Sunday significantly contribute to achieve good air quality, while the winter and the increasing flow of vehicles tend to worsen the air quality.

Keywords: particulates in the air; $\mathrm{MP}_{10}$; logistic model. 


\section{Introdução}

Estudos sobre a influência da poluição atmosférica na qualidade de vida do ser humano têm sido realizados com grande intensidade nos últimos anos (GUTJAHR, 2002; LEITE, 2005; MARTINS et al., 2002; PEITER; TOBAR, 1998; SCHAWRTZ; DOCKERY, 1992; SCHAWRTZ, 1999; GOUVEIA; FLETCHER, 2000; LIMA, 2001; FOKIANOS; KEDEM, 2004; MORETTIN; TOLOI, 2004; LEITE, 2005; LIMA et al., 2009). Estes trabalhos visaram avaliar os efeitos das variáveis que afetam a qualidade do ar, sendo que em alguns deles é feita uma relação direta entre a poluição do ar com as taxas de mortalidade e de doenças respiratórias.

A poluição atmosférica caracteriza-se basicamente pela presença de gases tóxicos e partículas sólidas no ar. As principais causas desse fenômeno são: a eliminação de resíduos por certos tipos de indústrias (siderúrgicas, petroquímicas, de cimento etc) e a queima de carvão e petróleo em usinas, automóveis e sistemas de aquecimento doméstico (LEITE, 2005; COOPER; ALLEY, 2002; THEODORE, 2008; SCHIFFTNER, 2002; WARK et al., 1998).

Nos grandes centros urbanos, tornam-se frequentes os dias em que o ar atinge níveis críticos, sejam pela ausência de ventos ou pelas inversões térmicas, que são períodos nos quais as correntes ascendentes do ar cessam, as quais são importantes para a limpeza dos poluentes acumulados nas camadas próximas à superfície.

Os efeitos causados ao meio ambiente e à saúde da população por causa da emissão destes poluentes, em especial dos materiais particulados, podem não ser apenas locais, pois dependem de fatores da região como, por exemplo, o relevo do entorno do ponto de emissão, as condições meteorológicas e a natureza dos poluentes. Isso significa que estes poluentes podem viajar milhares de quilômetros pela atmosfera, atingindo assim comunidades distantes do ponto de emissão.

Leite (2005) argumenta que a baixa umidade relativa e a baixa velocidade dos ventos são condições climáticas favoráveis para os altos níveis de poluentes encontrados nas grandes cidades; contudo, quando a precipitação pluviométrica e os ventos estão intensos, ocorre a dissipação dos poluentes do ar.

O crescimento das cidades, em muitos casos, desordenado e nãoplanejado faz com que os índices de poluição do ar fiquem próximos ao limite recomendado pela legislação ambiental e, em alguns casos, esses índices são ultrapassados, acarretando em risco à população da cidade.

A determinação sistemática da qualidade do ar, segundo o relatório de qualidade do ar da Cetesb (2009), deve ser limitada a um restrito número de poluentes, devido às questões de ordem prática e também em função de sua importância. De maneira geral, os poluentes consagrados universalmente como indicadores mais abrangentes da qualidade do ar são: monóxido de carbono, dióxido de enxofre, material particulado e ozônio.

A Resolução Conama 003/90 estabelece que, para as partículas menores que $10 \mu \mathrm{m}\left(\mathrm{MP}_{10}\right)$, a concentração média aritmética anual
(CMAA) não deve ultrapassar $50 \mu \mathrm{g}$ de pó $/ \mathrm{m}^{3}$ de ar amostrado, e a Cetesb (2008) estabelece classes de qualidade do ar de acordo com a concentração diária de $\mathrm{MP}_{10}$ no ar.

A cidade mineira de Uberlândia localiza-se na região do Triângulo Mineiro e possui uma população de mais de 600 mil habitantes, além de alto grau de desenvolvimento econômico, destacando-se a importância das agroindústrias. Esses fatores em conjunto são os principais propulsores da poluição do ar da região.

O conhecimento do comportamento temporal da poluição do ar pode contribuir com o planejamento das ações por parte dos órgãos ambientais, e uma das propostas para avaliar e estimar este comportamento temporal é por meio de análises estatísticas, como, por exemplo, séries temporais, análise de correlação e de regressão e análises multivariadas

A regressão logística é uma metodologia estatística que visa estimar probabilidades de ocorrências em variáveis dependentes do tipo binário.

Arango (2001) argumenta que a regressão logística, é uma ferramenta da estatística útil para situações nas quais se deseja predizer a presença ou ausência de uma determinada característica ou resultado, baseado em valores de um conjunto de variáveis independentes.

\section{Material e métodos}

As amostras para a avaliação do material particulado $\left(\mathrm{MP}_{10}\right)$, foram obtidas por um amostrador de grandes volumes para partículas respiráveis (AGV-MP 10 ), marca Thermo Andersen, modelo G10557PM10, instalado no Terminal Central de Ônibus Urbanos de Uberlândia, em Minas Gerais. O AGV-MP 10 foi instalado próximo ao cruzamento de duas avenidas, considerando-se o mínimo de interferências de barreiras físicas, considerou-se ainda o intenso tráfego de pessoas e veículos em torno da região. As medidas foram realizadas com periodicidade de três dias, sendo que cada coleta teve duração de 24 horas. Os cronogramas das amostragens e análises foram elaborados por discentes e docentes da Faculdade de Engenharia Química (FEQUI) da Universidade Federal de Uberlândia (UFU). Neste trabalho, foram utilizados os dados de concentração do $\mathrm{MP}_{10}$ de 2003 a 2008.

As partículas são coletadas em filtros de fibra de vidro. Estes filtros apresentam uma eficiência de coleta superior a 99\% para partículas com diâmetro aerodinâmico superior ou igual a 0,3 $\mu \mathrm{m}$. Antes de serem submetidos à amostragem, e após a realização destas, os filtros ficam no mínimo 24 horas em um ambiente condicionado (dessecador).

Após a determinação do $\mathrm{MP}_{10}$ no laboratório da FEQUI/UFU, os dados foram dispostos em planilhas eletrônicas, nas quais foram associados os dias da semana e a estação do ano em que foram realizadas as coletas.

A qualidade do ar foi classificada em "boa" e "não boa", tendo a CETESB (2008) considerado que a qualidade é boa, se o índice do $\mathrm{MP}_{10}$ for inferior a $50 \mu \mathrm{g} / \mathrm{m}^{3}$ e é não boa, se o $\mathrm{MP}_{10}$ for igual ou 
superior a $50 \mu \mathrm{g} / \mathrm{m}^{3}$. Para a aplicação dessas informações na equação logística, estes dados foram transformados em variável binária, sendo atribuído 1 para a classificação boa e 0 para não boa.

Foram associadas aos dados de concentração do $\mathrm{MP}_{10}$, as variáveis climáticas precipitação pluviométrica, temperatura média, umidade relativa e velocidade do vento, relativas ao período de 2003 a 2008, obtidas junto ao Instituto de Geografia (IG) da UFU, e também foram utilizadas as informações de fluxos de veículos no cruzamento das avenidas João Naves de Ávila com João Pinheiro, que se situam próximas ao ponto de coleta do $\mathrm{MP}_{10}$ e que foram obtidas na Faculdade de Engenharia Civil (FECIV).

As variáveis preditoras referentes ao clima (umidade relativa, velocidade do vento, precipitação diária e temperatura média) e ao fluxo de veículos foram utilizadas de forma contínua no modelo logístico. Já as variáveis relacionadas ao dia da semana e à estação do ano foram codificadas de forma binária, ou seja, atribuindo um para o dia da semana ou estação do ano em análise e zero para os demais. Desta forma, criaram-se sete variáveis binárias para dia da semana e quatro variáveis binárias para estação do ano.

O procedimento de análise estatística dos dados foi a determinação e interpretação do modelo de regressão logística simples. Foi utilizado o programa computacional BioEstat (AYRES et al., 2005) nas análises estatísticas dos dados.

Utilizou-se a equação logística simples que, de acordo com Ayres et al. (2005), é dada por:

$\operatorname{Logit}(\pi)=\beta_{0}+\beta_{1} X$ Equação 1

Onde:

Logit $(\pi)$ : função logística linearizada;

$\beta_{\mathrm{o}}$ :constante;

$\beta_{1}$ :coeficiente angular; $\mathrm{e}$

$\mathrm{X}$ : variável preditora.

As estimativas dos parâmetros da Equação 1 foram feitas com base nos dados amostrais e para o cálculo do Odds Ratio (OR), utilizou-se a Equação 2.

$\mathrm{OR}=\exp \left(\beta_{1}\right)$

Equação 2

No cálculo da probabilidade de ocorrência da variável dependente binária, dada uma condição da variável preditora, Ayres et al. (2005) indicam a seguinte função (Equação 3):

$$
P=\frac{1}{1+\exp \left(-\left(\beta_{0}+\beta^{1} X\right)\right)}
$$

Onde:

$P$ : probabilidade de ocorrência;

exp: base dos logaritmos neperianos;

$\beta_{0}$ : coeficiente linear ; $\beta_{1}$ : coeficiente angular; e

$\mathrm{X}$ : variável independente.

Para a verificação da significância dos atributos analisados na qualidade do ar, adotou-se o valor nominal de 10\%, ou seja, significâncias inferiores a 0,10 mostraram que as variáveis contribuem significativamente com a qualidade do ar.

\section{Resultados e discussão}

As estimativas de médias anuais do $\mathrm{MP}_{10}$, para a cidade de Uberlândia, em Minas Gerais, no período de 2003 a 2008, são apresentadas na Tabela 1. O maior valor do $\mathrm{MP}_{10}$ ocorreu em 2004, entretanto, pode-se observar que a amplitude de variação ao longo dos anos é relativamente pequena. Em 2004, 2005 e 2008, a média anual ultrapassou o limite de $50 \mu \mathrm{g} / \mathrm{m}^{3}$ e nos demais anos, a média ficou próxima deste índice. Segundo a Resolução Conama 03/90, ultrapassar este limite significa as condições que podem afetar a saúde da população e o meio ambiente. A média do período analisado para a cidade de Uberlândia foi de $50,26 \mu \mathrm{g} / \mathrm{m}^{3}$. Comparando este resultado com os valores obtidos de cidades do mesmo porte, como é o caso de São José dos Campos (622 mil habitantes) e Ribeirão Preto (563 mil habitantes) do interior do estado de São Paulo, observa-se que o nível de concentração de particulados $\left(\mathrm{MP}_{10}\right)$ em Uberlândia é superior àquele destas cidades. Ribeirão Preto apresentou valores médios, no mesmo período, de 40,25 $\mu \mathrm{g} / \mathrm{m}^{3} \mathrm{e}$ São José dos Campos de 26,17 $\mu \mathrm{g} / \mathrm{m}^{3}$ (CETESB, 2009).

Será apresentada a seguir a aplicação de modelos logísticos a cada uma das variáveis estudadas, com os respectivos impactos na qualidade do ar. Foram apresentadas, para cada variável, simulações da qualidade do ar a partir de níveis arbitrados dessas variáveis.

$\mathrm{Na}$ Tabela 2, são apresentados os resultados da equação logística da qualidade do ar em função da umidade relativa e precipitação pluviométrica.

Os resultados mostraram que os elementos climáticos, a umidade relativa e a precipitação interferem significativamente na quantidade de $\mathrm{MP}_{10}$ presente no ar, favorecendo ou não a poluição atmosférica como mostra a Tabela 2.

Como a significância da umidade relativa e da precipitação está abaixo de 0,10, verifica-se que essas duas variáveis meteorológicas possuem relação com a qualidade do ar avaliada por meio de $\mathrm{MP}_{10}$.

Usando a Equação 1 e considerando-se a umidade relativa do ar de $40 \%$, tem-se: Logit $\pi=-0,908$, portanto $P$ (Equação 3), que é a probabilidade da qualidade do ar ser boa, é igual a 28,74\%. De forma semelhante, para a umidade relativa de $90 \%$, tem-se Logit $\pi=1,842$ e $P=86,32 \%$, significando que quanto maior a umidade relativa, melhor a qualidade do ar

O mesmo cálculo pode ser feito para a precipitação. Na condição de precipitação zero, tem-se $56,41 \%$ de chance de se obter boa qualidade do ar, já para um dia com precipitação de $10 \mathrm{~mm}$, tem-se $64,29 \%$ de probabilidade de qualidade boa do ar. 
Analisando o OR (exp $(\beta))$ das equações apresentadas na Tabela 2 , verifica-se que, para cada unidade de acréscimo na umidade relativa, espera-se, em média, 5,6\% de acréscimo na probabilidade da qualidade do ar ser considerada boa e para cada $1 \mathrm{~mm}$ de precipitação, espera-se acréscimo de 3\% na probabilidade da qualidade do ar ser boa. Os intervalos de confiança revelam a amplitude do OR com confiança de $90 \%$.

$\mathrm{Na}$ análise da qualidade do ar em função dos dias da semana em que se coletaram os dados (Tabela 3), verificou-se que apenas sábado e domingo possuem a relação significativa.

A partir dos dados de OR $(\exp (\beta))$ da Tabela 3, pode-se concluir que, no domingo, tem-se 2,194 vezes mais chances de ter qualidade boa do ar em relação aos demais dias da semana, já com relação ao sábado, essa chance foi de três vezes.

Analisando-se ainda os intervalos de confiança dos OR, foi possível verificar que sábado e domingo possuem estatisticamente a mesma chance de ter qualidade boa do ar, pois os intervalos apresentam interseção.

Utilizando as equações logísticas (Equação 1), tem-se que, no sábado, a probabilidade de qualidade do ar boa é de 79,24\% e, para o domingo, essa probabilidade é de 75,00\%.

A Tabela 4 mostra a relação entre as estações do ano e a qualidade do ar atmosférico para as estações que se apresentaram estatisticamente significantes, ou seja, para o verão, o inverno e a primavera. $\mathrm{O}$ outono apresentou-se não-significativo, ou seja, não é possível utilizar a equação logística para o cálculo das probabilidades nesta estação.

Os valores do OR apresentados na Tabela 4 mostram que a chance de qualidade boa no verão e na primavera é de 2,6 e de 1,6, respectivamente. Considerando-se o inverno, a chance é de 0,248 vezes.
Estes resultados mostram claramente o cuidado que deve ser tomado nesta estação devido aos níveis baixos de qualidade do ar.

Aplicando-se as informações das estações do ano em cada equação logística, foi obtido que, no verão, a probabilidade de qualidade boa do ar é de 75,26\%, na primavera é de 68,07\% e no inverno, de $34,63 \%$

Os resultados apresentados seguem as observações encontradas em Leite (2005), Vieira Neto et al. (2008), entre outros, os quais argumentam que o aumento da precipitação e da umidade relativa do ar, assim como estações do ano com maior pluviosidade e dias de fins de semana, proporcionam maiores chances de serem obtidas melhores qualidade do ar.

O fluxo de veículos também possui relação com a qualidade do ar, pois a sua significância está abaixo de 0,10 como mostra a Tabela 5 .

O valor do OR mostra que o aumento do fluxo de veículos na região central de Uberlândia, reduz a probabilidade de se obter boa qualidade do ar.

Analisando a equação logística, tem-se que, para um fluxo de 4.000 veículos por dia no cruzamento das avenidas João Pinheiro com João Naves de Ávila, a probabilidade de boa qualidade do ar é de 74\% e para fluxo de 20.000 veículos tem-se 36,5\% de probabilidade, fato este que pode estar relacionado com a melhor qualidade do ar nos finais de semana. Este resultado explica a melhor qualidade do ar no fim de semana, no qual a movimentação de veículos é menos intensa.

Foi possível observar também que os valores de $\pi$ da Equação 1 para o fluxo de veículos, foi um dos mais elevados dentre as variáveis analisadas, sendo inferior apenas ao inverno. Estes resultados mostram

Tabela 1 - Estimativas de médias anuais para $\mathrm{MP}_{10}\left(\mu \mathrm{g} / \mathrm{m}^{3}\right)$ na cidade de Uberlândia - MG

\begin{tabular}{llllll} 
Ano & 2003 & 2004 & 2005 & 2006 & 2007 \\
Média $\left(\mu \mathrm{g} / \mathrm{m}^{3}\right)$ & 47,98 & 55,09 & 52,42 & 46,64 & 2008 \\
\hline
\end{tabular}

Tabela 2 - Equação logística da qualidade do ar em função da umidade relativa e precipitação

\begin{tabular}{lcccccccc} 
& B & S.E. & Wald & df & Sig. & Exp (B) & \multicolumn{2}{c}{$\begin{array}{c}90 \% \text { Cl para EXP(B) } \\
\text { Abaixo }\end{array}$} \\
U. RELATIVA & 0,055 & 0,009 & 40,637 & 1 & 0,000 & 1,056 & 1,041 \\
Constante & $-3,108$ & 0,554 & 31,509 & 1 & 0,000 & 0,045 & 1,071 \\
Precipitação & 0,033 & 0,014 & 5,730 & 1 & 0,017 & 1,033 & 1,010 \\
Constante & 0,258 & 0,114 & 5,156 & 1 & 0,023 & 1,295 & 1,057
\end{tabular}

B: estimativas dos parâmetros das equações; SE: erro padrão; Wald: estatística de Wald; df: graus de liberdade; Sig: significância da estatística de Wald; Exp (B): OR; 90\% Cl: intervalo de confiança de $90 \%$ para OR; U.Relativa: Umidade Relativa.

Tabela 3 - Equação logística da qualidade do ar em função de sábado e domingo

\begin{tabular}{lcccccccc} 
& B & S.E & Wald & df & Sig. & Exp (B) & \multicolumn{2}{c}{ A0\% C.I para EXP(B) } \\
DOMINGO & 0,786 & 0,422 & 3,460 & 1 & 0,063 & 2,194 & 1,095 & 4,395 \\
Constante & 0,313 & 0,109 & 8,311 & 1 & 0,004 & 1,367 & \\
SÁBADO & 1,100 & 0,357 & 9,520 & 1 & 0,002 & 3,004 & 1,671 & 5,401 \\
Constante & 0,240 & 0,111 & 4,629 & 1 & 0,031 & 1,271 &
\end{tabular}

B: estimativas dos parâmetros das equações; SE: erro padrão; Wald: estatística de Wald; df: graus de liberdade; Sig: significância da estatística de Wald; Exp (B): OR; 90\% Cl: intervalo de confiança de $90 \%$ para OR. 
que o intenso fluxo de veículos na estação do inverno é uma condição extremamente crítica para a qualidade do ar da cidade de Uberlândia.

Após a análise das variáveis que contribuíram significativamente para a qualidade do ar ter sido concluída, serão apresentados os resultados das variáveis não-significativas ( $p>0,10)$. A Tabela 6 mostra os resultados obtidos a partir da regressão logística para temperatura, velocidade do vento, segunda-feira, terça-feira, quarta-feira, quinta-feira, sexta-feira e o outono, que não afetaram significativamente a qualidade do ar.

\section{Conclusões}

Este trabalho permitiu concluir que existe relação significativa de atributos climáticos e variáveis temporais com a qualidade do ar de
Uberlândia. Além disso, modelos logísticos simples podem ser usados para calcular probabilidades de se obter qualidade considerada boa do ar. Os modelos logísticos simples mostraram que a precipitação, a umidade relativa, o verão, a primavera, o sábado e o domingo contribuem significativamente para se obter qualidade do ar boa, enquanto o inverno e o fluxo de veículos crescente tendem a reduzir a probabilidade de se obter boa qualidade do ar.

\section{Agradecimentos}

À Fundação de Amparo à Pesquisa do Estado de Minas Gerais (FAPEMIG) pelo aporte de recursos financeiros e bolsa de iniciação científica.

Tabela 4 - Equação logística da qualidade do ar em função do verão, primavera e inverno

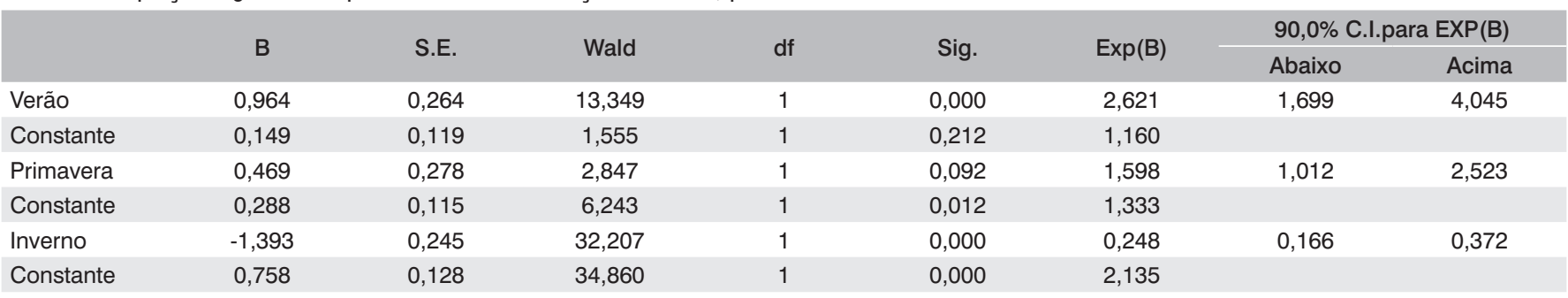

B: estimativas dos parâmetros das equações; SE: erro padrão; Wald: estatística de Wald; df: graus de liberdade; Sig: significância da estatística de Wald; Exp (B): OR; 90\% Cl: intervalo de confiança de $90 \%$ para OR.

Tabela 5 - Equação logística da qualidade do ar em função do fluxo de veículos

\begin{tabular}{lcccccccc} 
& B & S.E. & Wald & df & Sig. & Exp(B) & \multicolumn{2}{c}{ 90\% C.I.para EXP(B) } \\
Fluxo de veículos & $-0,099$ & 0,035 & 7,993 & 1 & 0,005 & 0,906 & 0,855 & 0,960 \\
Constante & 1,425 & 0,397 & 12,892 & 1 & 0,000 & 4,157 &
\end{tabular}

B: estimativas dos parâmetros das equações; SE: erro padrão; Wald: estatística de Wald; df: graus de liberdade; Sig: significância da estatística de Wald; Exp (B): OR; $90 \%$ Cl: intervalo de confiança de $90 \%$ para OR.

Tabela 6 - Equação logística da qualidade do ar em função da temperatura média, velocidade do vento, de segunda-feira, de terça-feira, de quarta-feira, de quinta-feira, de sexta-feira e do Outono

\begin{tabular}{|c|c|c|c|c|c|c|c|c|}
\hline & \multirow{2}{*}{ B } & \multirow{2}{*}{ S.E. } & \multirow{2}{*}{ Wald } & \multirow{2}{*}{ df } & \multirow{2}{*}{ Sig. } & \multirow{2}{*}{$\operatorname{Exp}(B)$} & \multicolumn{2}{|c|}{$90,0 \%$ C.I.para EXP(B) } \\
\hline & & & & & & & Abaixo & Acima \\
\hline TEMPERATURA & $-0,054$ & 0,043 & 1,566 & 1 & 0,211 & 0,948 & 0,883 & 1,017 \\
\hline Constante & 1,662 & 1,037 & 2,568 & 1 & 0,109 & 5,272 & & \\
\hline SEGUNDA-FEIRA & $-0,180$ & 0,278 & 0,417 & 1 & 0,518 & 0,836 & 0,529 & 1,320 \\
\hline Constante & 0,403 & 0,115 & 12,353 & 1 & 0,000 & 1,496 & & \\
\hline TERÇA-FEIRA & $-0,437$ & 0,286 & 2,325 & 1 & 0,127 & 0,646 & 0,403 & 1,035 \\
\hline Constante & 0,437 & 0,114 & 14,598 & 1 & 0,000 & 1,548 & & \\
\hline QUARTA-FEIRA & $-0,370$ & 0,279 & 1,759 & 1 & 0,185 & 0,691 & 0,437 & 1,093 \\
\hline Constante & 0,434 & 0,115 & 14,314 & 1 & 0,000 & 1,544 & & \\
\hline QUINTA-FEIRA & $-0,272$ & 0,319 & 0,727 & 1 & 0,394 & 0,762 & 0,451 & 1,287 \\
\hline Constante & 0,405 & 0,112 & 13,218 & 1 & 0,000 & 1,500 & & \\
\hline SEXTA-FEIRA & $-0,249$ & 0,281 & 0,784 & 1 & 0,376 & 0,780 & 0,491 & 1,238 \\
\hline Constante & 0,413 & 0,114 & 13,058 & 1 & 0,000 & 1,512 & & \\
\hline OUTONO & 0,153 & 0,232 & 0,436 & 1 & 0,509 & 1,165 & 0,796 & 1,706 \\
\hline Constante & 0,329 & 0,123 & 7,106 & 1 & 0,008 & 1,389 & & \\
\hline
\end{tabular}

B: estimativas dos parâmetros das equações; SE: erro padrão; Wald: Estatística de Wald; df: graus de liberdade; Sig: significância da estatística de Wald; Exp (B): OR; 90\% Cl: intervalo de confiança de $90 \%$ para OR. 


\section{Referências}

ARANGO, H. G. Bioestatística: Teórica e Computacional. Rio de Janeiro: Guanabara Koogan, 2001, 440p.

AYRES, M.; AYRES Jr, M.; AYRES, D. L.; dos SANTOS, A. S. BioEstat 4.0: Aplicações estatísticas nas áreas das ciências biológicas e médicas. Belém: Sociedade Civil Mamirauá; Brasília: CNPq, 2005, 324p.

COMPANHIA DE TECNOLOGIA DE SANEAMENTO AMBIENTAL (CETESB). Secretaria do Estado do Meio Ambiente de São Paulo. Relatório de Qualidade do Ar no Estado de São Paulo/2009. Disponível em: http://www.cetesb.sp.gov.br/Ar/publicacoes.asp. Acessado em: 15 de Janeiro de 2011.

Padrões de Qualidade do ar. Disponível em: http://www.cetesb. sp.gov.br/Ar/ar_indice_padroes.asp. Acessado em: 14 de Dezembro de 2008.

COOPER, C. D.; ALLEY, F. C. Air pollution control: A design approach. Long Grove, Illinois: Waveland Press, Inc, 2002, 738 p.

FOKIANOS, K.; KEDEM, B. Partial likelihood inference for time series following generalized linear models, Jounal of Time Series Analysis, Oxford, v.25, n. 2, p.173-197, 2004

GOUVEIA, N.; FLETCHER, T. Time series analysis of air pollution and mortality: effects by cause, age and socioeconomic status. Epidemiology Community Health, London, v. 54, n. 10, p.750-755, 2000.

GUTJAHR, M. R. A Poluição do ar em Paulínia - SP: Uma análise históricogeográfica do clima. 2002, 239p. Tese (Doutorado em Geografia Física). Pós-graduação em Geografia Física. Universidade de São Paulo - São Paulo- SP.

LEITE, N. O. Modelagem estatística da relação entre poluição atmosférica e mortalidade por doenças respiratórias. 2005, 64 p. Dissertação (Mestrado em Estatística e Experimentação Agropecuária) - Universidade Federal de Lavras, Lavras, MG, 2005

LIMA, E. A. P.; GUIMARÃES, E. C.; POZZA, S. A.; BARROZO, M. A. S; COURY, J. R. A study of atmospheric particulate matter in a city of the central region of Brazil using time-series analysis, International Journal of Environmental Engineering, 2009, v. 1, n.1, p. 80-94, 2009

LIMA, L. P. Modelos aditivos generalizado: aplicação a um estudo epidemiológico ambiental. Dissertação (Mestrado em Estatística) Universidade de São Paulo, São Paulo, SP, 2001.

MARTINS, L. C. et al. Poluição atmosférica e atendimentos por pneumonia e gripe em São Paulo, Brasil, Revista Saúde Pública, v. 36 , n. 1, p. 88-94, 2002

MORETTIN, P. A.; TOLOI, C. M. C. Análise de séries temporais. São Paulo: Edagrd Blucher, 2004, $535 \mathrm{p}$

PEITER, P.; TOBAR, C. Poluição do ar e condições de vida: uma análise geográfica de riscos à saúde em Volta Redonda, Rio de Janeiro, Brasil, Cadernos de Saúde Pública, v. 14, n. 3, pp. 473-485, 1998.

SCHIFFTNER, K. C. Air pollution control equipment selection guide. Lewis Publishers, 2002, $228 \mathrm{p}$

SCHWARTZ, J. Air pollution and daily mortality in Birmingham, Alabama, American Journal of Epidemiology, Baltimore, v. 137, n.10, p.1136-1147 1999

SCHWARTZ, J.; DOCKERY, D. W. Particulate air pollution and daily mortality in Steubenville, Ohio, American Journal of Epidemiology, Baltimore, v. 135, n.1, p.12-19, 1992.

THEODORE, L. Air pollution control equipment calculations. Hoboken, New Jersey: John Wiley \& Sons, Inc, 2008, 574 p.

VIEIRA NETO, J. F; GUIMARÃES, E. C.; BARROZO, M. A. S.; LIMA, E. A. P.; TAVARES, M. Análise de correlações entre atributos climáticos e poluição atmosférica em Uberlândia - MG, Horizonte Científico, v. 1, n. 8. 16 p. 2008. Disponível em: http://www.horizontecientifico.propp.ufu. br. Acessado em 12 de fevereiro de 2009.

WARK, K.; WARNER, C. F.; DAVIS, W. T. Air pollution: Its origin and control. Addison Wesley Longman, Inc, $3^{\text {rd }}$ ed., 1998, 573 p. 